\title{
Analysis of the Current Status and Influencing Factors of the Readiness for Discharge of Patients after Prostate Cancer Surgery Based on the Concept of Enhanced Recovery after Surgery (ERAS)
}

\author{
Youdi Cai', Limei Zhang1, Kun Bao1, Huan Jian'1, Jinhong Zhong1, Yuanyuan Huang1, Limin Liang2* \\ ${ }^{1}$ Department of Urological Surgery, The Third Affiliated Hospital of Sun Yat-sen University, Guangzhou, China \\ ${ }^{2}$ Department of Cardio-Thoracic Surgery, The Third Affiliated Hospital of Sun Yat-sen University, Guangzhou, China \\ Email: 417192990@qq.com, *45676168@qq.com
}

How to cite this paper: Cai, Y.D., Zhang, L.M., Bao, K., Jian, H., Zhong, J.H., Huang, Y.Y. and Liang, L.M. (2021) Analysis of the Current Status and Influencing Factors of the Readiness for Discharge of Patients after Prostate Cancer Surgery Based on the Concept of Enhanced Recovery after Surgery (ERAS). Open Journal of Nursing, 11, 10861097.

https://doi.org/10.4236/ojn.2021.1112086

Received: November 30, 2021

Accepted: December 27, 2021

Published: December 30, 2021

Copyright $\odot 2021$ by author(s) and Scientific Research Publishing Inc. This work is licensed under the Creative Commons Attribution International License (CC BY 4.0).

http://creativecommons.org/licenses/by/4.0/

(c) (i) Open Access

\begin{abstract}
Objective To investigate the level of readiness for discharge of patients after prostate cancer surgery based on the concept of Enhanced Recovery After Surgery (ERAS), and to explore its influencing factors, so as to provide references for improving the readiness for discharge of patients after prostate cancer surgery. Methods The general information questionnaire, the discharge preparation scale, and the discharge guidance quality scale were used to investigate 119 patients discharged from the urological surgery department of a tertiary A-level hospital in Guangzhou after radical prostatectomy. Results The total score of discharge readiness of patients after radical prostatectomy was 147.74 \pm 35.71 points, which was at a lower middle level and the total score of discharge guidance quality was $180.68 \pm 38.91$ points, which was at a medium level. Multiple linear regression analysis showed that education level, family monthly income, Gleason score, whether to perform lymphatic dissection, whether to discharge with a urinary catheter, and the quality of discharge guidance were the main factors influencing the readiness for discharge of patients after prostate cancer surgery. Conclusion In clinical nursing work, it is necessary to implement individualized health education according to the characteristics and needs of different patients to improve the level of preparation for discharge of patients after prostate cancer surgery.
\end{abstract}

\section{Keywords}

Prostate Cancer, Readiness for Discharge, Quality of Discharge Guidance, Analysis of Influencing Factors 


\section{Introduction}

Prostate cancer is a common urinary system tumor in men; in recent years, the incidence of prostate cancer has gradually increased in China, and the recurrence rate and mortality rate are relatively high [1]. For patients with localized prostate cancer, radical surgery is the preferred treatment. However, surgical treatment may cause loss of reproductive organs, sexual dysfunction, urinary incontinence, etc., which will affect the physiology and psychology of patients to varying degrees [2] [3]. Some researchers [4] pointed out that after getting discharged from hospital, patients with prostate cancer are still in high demands of guidance on aspects such as follow-up treatment options, medication instructions, urinary control rehabilitation exercises, sexual function recovery, and psychological and social support. The concept of discharge readiness was proposed by Fenwick et al., referring to the comprehensive analysis of the patient's physiological, psychological, and social conditions by medical staff to determine whether the patient has the ability to leave the hospital and return to society for rehabilitation; it is a type of prediction for the safety of the patient during the transitional period after discharge [5]. Patients undergoing prostate cancer surgery under the management of the Enhanced Recovery After Surgery (ERAS) model can be discharged after their physiological functions have basically returned to normal and have a certain degree of self-care ability, whether the patients themselves are truly ready for discharge and subsequent recovery has not been considered. Therefore, it is very important to effectively evaluate the patient's preparedness before discharge. However, there are few domestic and foreign related studies on the readiness for postoperative discharge of prostate cancer patients under the concept of ERAS [6]. This study analyzes the readiness for discharge of patients with prostate cancer after surgery and explores its influencing factors. It aims to improve the readiness of patients for discharge, ensure the safety of patients after discharge, and improve the self-care ability of patients after discharge. The report is as follows.

\section{Subjects and Methods}

\subsection{Research Subjects}

119 patients who underwent radical prostatectomy in our hospital from April 2020 to August 2021 were selected as the research subjects. Inclusion criteria: 1) Patients diagnosed as prostate cancer through prostate puncture and radical prostatectomy was performed; 2) Informed consent of patients was obtained; 3 ) Discharged patients who have reached the discharge standard after inpatient treatment, such as walk freely, be painless with oral analgesics, resume half-flow diet and have no risk of complications; 4) Patients able to use Mandarin or Cantonese proficiently, and able to communicate effectively with the investigators. Exclusion criteria: 1) Patients with consciousness disorders; 2) Patients with mental disorders; 3) Patients with other tumors; 4) Patients with other serious physical diseases and can't finish the investigation. 


\subsection{Methods}

\subsubsection{Survey Tools}

1) General information questionnaire: self-designed questionnaire was used, which contained demographic data and disease data. Demographic data mainly included age, education level, marital status, working status, monthly family income, medical payment method, place of residence, main caregivers, etc. Disease data mainly included Gleason score, operation method, whether to perform lymphatic dissection, postoperative hospital stay, and whether to discharge with a urinary catheter.

2) Readiness Hospital Discharge Scale (RHDS) [7]: it is a self-rating scale developed by researchers such as Weiss and Piacentine. Patients were evaluated on the day of discharge. The scale contains 4 dimensions and 23 items in total: physical condition ( 7 items), disease knowledge (8 items), coping ability after discharge (3 items), expected social support after discharge (4 items), and 1 additional item that is not included in the scoring system (true-false item). The total score of the scale is $0-220$ points; the higher the score, the better the patient's readiness for discharge. The scale is widely used abroad to assess the readiness for discharge of patients with different diseases. The Cronbach's $\alpha$ coefficient of the entire scale ranges from 0.84 to 0.92 in different populations, showing good reliability. This study used the Chinese version of RHDS [8] translated and revised by Zhao Huiling of West China Hospital of Sichuan University. The total Cronbach's $\alpha$ coefficient of the scale was 0.929 , and the Cronbach's $\alpha$ coefficient of each dimension was $0.824-0.901$.

3) Quality of Discharge Teaching Scale (QDTS) [9]: This scale includes 3 dimensions: required content (what the patient needs before discharge, 6 items), obtained content (what is actually obtained, 6 items), and the guidance skills and effects (12 items). Each item is scored from 0 to 10 points. The Cronbach's $\alpha$ coefficient of the overall scale was 0.92 ; the Cronbach's $\alpha$ coefficient of the sub-scales (required content, obtained content, guidance skills and effects) were $0.85,0.88$ and 0.93 , respectively. The quality of discharge guidance was measured by calculating the total score of the two dimensions of "required content" and "guidance skills and effects". The higher the total score, the better the quality of guidance.

\subsubsection{Sample Size Calculation}

The sample size was at least $\mathrm{N} \times 5$, where $\mathrm{N}$ was the number of items, and $10 \%$ $20 \%$ of unrecovered and invalid questionnaires were considered. The total sample size was then at least $(\mathrm{N} \times 5) \times(1+10) \%$ cases. According to this study, the preset number of entry items was 23 , and therefore 126 samples were required.

\subsubsection{Data Collection}

The consent and support of the leaders of the relevant departments were firstly obtained. Before issuing the questionnaire, the purpose and significance of this research were explained to the respondents; following the principle of volunta- 
riness, the questionnaires were anonymously filled. The inclusion and exclusion criteria of the samples were strictly followed, and the questionnaire surveys were conducted on the selected subjects on the day when the patient was discharged from the hospital. Unified instruction and form-filling methods were utilized to explain how to fill in the questionnaire; for patients who were illiterate or had limitation in activities, the investigator would read each item for the patients, and fill out the questionnaire based on the patients' answers. After the patients completed the questionnaire, the investigators would carefully check the questionnaire and fill in on the spot if there were vacancies or omissions. In this study, a total of 130 questionnaires were distributed and 119 valid questionnaires were returned. The effective response rate was $92 \%$.

\subsubsection{Statistical Analysis}

The data was entered into Excel to establish a database, and SPSS23.0 software was used for statistical analysis. The general information of patients was described by frequency, mean and standard deviation. The sample size of this study was relatively large, which could be regarded as approximately normally distributed data. The dimensions of the scale and the score of the total scale were described by the mean \pm standard deviation. In univariate analysis, two sets of independent sample t-tests were used for binary categorical variables; single-factor analysis of variance was used for multi-categorical variables that met the homogeneity of variance, and otherwise non-parametric tests (Kruskal-Wallis $\mathrm{H}$ test) were used. For Pearson correlation analysis, multiple linear regression was utilized for multivariate analysis.

\section{Results}

\subsection{Readiness for Discharge after Prostate Cancer Surgery}

Since the number of items in each dimension of the scale was different and the range of scores was inconsistent, the dimension scores were standardized: standardized score $=$ dimension total score/number of items. According to item 1, 114 patients (95.80\%) thought they were ready for discharge, and the other 5 patients (4.20\%) thought they were not ready. The total score of readiness for discharge of patients after prostate cancer surgery was (147.74 \pm 35.71$)$ points, and the scores of each dimension were shown in Table 1.

Table 1. Discharge readiness scores of patients with prostate cancer surgery $(\mathrm{n}=119$, points, $\bar{x} \pm s)$.

\begin{tabular}{cccccc}
\hline Item & $\begin{array}{c}\text { Physical } \\
\text { condition } \\
(7)\end{array}$ & $\begin{array}{c}\text { Disease } \\
\text { knowledge } \\
(8)\end{array}$ & $\begin{array}{c}\text { Coping } \\
\text { ability } \\
\text { after } \\
\text { discharge (3) }\end{array}$ & $\begin{array}{c}\text { Social } \\
\text { support } \\
\text { after } \\
\text { discharge (4) }\end{array}$ & $\begin{array}{c}\text { Total } \\
\text { score } \\
(22)\end{array}$ \\
\hline Actual score & $42.92 \pm 11.29$ & $53.55 \pm 16.38$ & $20.31 \pm 5.97$ & $30.96 \pm 7.47$ & $147.74 \pm 35.71$ \\
Standard score & $6.13 \pm 1.61$ & $6.69 \pm 2.05$ & $6.77 \pm 1.99$ & $7.74 \pm 1.87$ & $6.72 \pm 1.62$ \\
\hline
\end{tabular}


3.2. The Quality of Discharge Guidance for Patients after Prostate Cancer Surgery, as Shown in Table 2

Table 2. Discharge guidance quality scores of patients after prostate cancer surgery $(\mathrm{n}=$ 119 , points, $\bar{x} \pm s)$.

\begin{tabular}{ccccc}
\hline Dimension & $\begin{array}{c}\text { Number of } \\
\text { items }\end{array}$ & Full score & Actual score & $\begin{array}{c}\text { Standardized } \\
\text { score }\end{array}$ \\
\hline Required content & 6 & 60 & $42.31 \pm 12.84$ & $7.05 \pm 2.14$ \\
Obtained content & 6 & 60 & $41.64 \pm 12.81$ & $6.94 \pm 2.14$ \\
Guidance skills and effects & 12 & 120 & $96.73 \pm 18.15$ & $8.06 \pm 1.51$ \\
Total score & 24 & 240 & $180.68 \pm 38.91$ & $7.53 \pm 1.62$ \\
\hline
\end{tabular}

\subsection{Univariate Analysis of Readiness for Discharge of Patients after Prostate Cancer Surgery, See Table 3 for Details}

Table 3. Comparison of discharge readiness scores of patients with prostate cancer surgery among different factor groups $(\mathrm{n}=119)$.

\begin{tabular}{|c|c|c|c|c|}
\hline Item & $\begin{array}{c}\text { Number of } \\
\text { cases }\end{array}$ & $\begin{array}{l}\text { Total score of discharge } \\
\text { readiness (points, } \bar{x} \pm s \text { ) }\end{array}$ & $\begin{array}{l}\text { Statistical } \\
\text { quantity }\end{array}$ & $P$ value \\
\hline \multicolumn{5}{|l|}{ Age (years) } \\
\hline$<65$ & 33 & $157.24 \pm 22.54$ & 1.816 & 0.072 \\
\hline$\geq 65$ & 86 & $144.09 \pm 39.12$ & & \\
\hline \multicolumn{5}{|l|}{ Education level } \\
\hline Below junior high school & 65 & $135.48 \pm 38.45$ & -4.421 & $<0.001$ \\
\hline Above high school & 54 & $162.50 \pm 25.44$ & & \\
\hline \multicolumn{5}{|l|}{ Marital status } \\
\hline Married & 114 & $147.46 \pm 36.16$ & -0.412 & 0.681 \\
\hline No spouse & 5 & $154.20 \pm 25.15$ & & \\
\hline \multicolumn{5}{|l|}{ Working status } \\
\hline On-the-job & 18 & $143.72 \pm 21.01$ & -0.516 & 0.606 \\
\hline Retired & 101 & $148.46 \pm 37.76$ & & \\
\hline \multicolumn{5}{|l|}{ Monthly family income } \\
\hline$<6000$ & 71 & $140.93 \pm 40.43$ & -2.591 & 0.011 \\
\hline$\geq 6000$ & 48 & $157.81 \pm 24.35$ & & \\
\hline \multicolumn{5}{|l|}{ Medical payment method } \\
\hline Without reimbursement & 11 & $137.27 \pm 46.65$ & -1.021 & 0.310 \\
\hline With reimbursement & 108 & $148.81 \pm 34.50$ & & \\
\hline \multicolumn{5}{|l|}{ Place of residence } \\
\hline Urban & 83 & $152.06 \pm 32.29$ & 2.031 & 0.045 \\
\hline Rural & 36 & $137.78 \pm 41.34$ & & \\
\hline \multicolumn{5}{|l|}{ Main caregivers } \\
\hline Spouse & 85 & $148.65 \pm 38.19$ & 0.437 & 0.663 \\
\hline Children & 34 & $145.47 \pm 28.99$ & & \\
\hline
\end{tabular}




\section{Continued}

\begin{tabular}{|c|c|c|c|c|}
\hline Gleason score & & & \multirow{3}{*}{3.463} & \multirow{3}{*}{0.001} \\
\hline$<7$ & 66 & $157.45 \pm 27.46$ & & \\
\hline$\geq 7$ & 53 & $135.64 \pm 41.01$ & & \\
\hline \multicolumn{5}{|l|}{ Operation method } \\
\hline Laparoscopy & 44 & $148.27 \pm 31.49$ & \multirow[t]{2}{*}{0.124} & \multirow[t]{2}{*}{0.901} \\
\hline Robot assisted surgery & 75 & $147.43 \pm 38.17$ & & \\
\hline \multicolumn{3}{|l|}{$\begin{array}{l}\text { Whether to perform } \\
\text { lymphatic dissection }\end{array}$} & \multirow{3}{*}{-3.574} & \multirow{3}{*}{0.001} \\
\hline Yes & 43 & $132.91 \pm 44.00$ & & \\
\hline No & 76 & $156.13 \pm 26.92$ & & \\
\hline \multicolumn{3}{|l|}{$\begin{array}{c}\text { Postoperative hospital } \\
\text { stay (days) }\end{array}$} & \multirow{3}{*}{0.739} & \multirow{3}{*}{0.462} \\
\hline$\leq 7$ & 89 & $149.15 \pm 36.33$ & & \\
\hline$>7$ & 30 & $143.57 \pm 34.03$ & & \\
\hline \multicolumn{5}{|l|}{ Whether to discharge } \\
\hline with urinary catheter & & & \multirow{3}{*}{-3.525} & \multirow{3}{*}{0.001} \\
\hline Yes & 43 & $133.09 \pm 39.72$ & & \\
\hline No & 76 & $156.03 \pm 30.50$ & & \\
\hline \multicolumn{5}{|l|}{ Quality of discharge } \\
\hline guidance & & & \multirow{3}{*}{-3.262} & \multirow{3}{*}{0.001} \\
\hline Good & 111 & $150.50 \pm 34.21$ & & \\
\hline General & 8 & $109.50 \pm 36.29$ & & \\
\hline
\end{tabular}

\subsection{Analysis of the Correlation between the Discharge Readiness of Patients with Prostate Cancer Surgery and the Quality of Discharge Guidance}

The results of this study showed that the hospital discharge readiness of patients after radical prostatectomy was positively correlated with the quality of discharge guidance $(r=0.140)$. The correlation between discharge readiness and the scores of various dimensions of guidance quality was shown in Table 4.

\subsection{Multiple Linear Regression Analysis of Factors Influencing the Readiness for Discharge of Patients with Prostate Cancer Surgery}

Taking the total score of hospital discharge readiness (y1) as the dependent variable, the independent variables including education level ( $\mathrm{x} 1)$, monthly family income (x2), place of residence ( $\mathrm{x} 3$ ), Gleason score ( $\mathrm{x} 4)$, whether to perform lymphatic dissection $(\mathrm{x} 5)$, whether to discharge with urinary catheter $(\mathrm{x} 6)$, discharge guidance ( $\mathrm{y} 2$ as the actual evaluation value), etc. were put into the equation for multiple linear regression analysis. The results showed that the education level, family monthly income, Gleason score, whether to perform lymphatic dissection, whether to discharge with urinary catheter, and discharge guidance were all related to the patient's readiness for discharge, and the difference was statistically significant $(\mathrm{P}<0.05)$, see Table 5 . 
Table 4. Correlation analysis of discharge readiness and discharge guidance quality of patients with prostate cancer surgery $(\mathrm{n}=$ $119, \mathrm{r}$ value).

\begin{tabular}{|c|c|c|c|c|c|}
\hline Dimension & $\begin{array}{c}\text { Total score of } \\
\text { discharge readiness }\end{array}$ & $\begin{array}{l}\text { Physical } \\
\text { condition }\end{array}$ & $\begin{array}{c}\text { Disease } \\
\text { knowledge }\end{array}$ & $\begin{array}{l}\text { Coping ability } \\
\text { after discharge }\end{array}$ & $\begin{array}{l}\text { Social support } \\
\text { after discharge }\end{array}$ \\
\hline $\begin{array}{c}\text { Total score of } \\
\text { discharge guidance quality }\end{array}$ & 0.140 & -0.011 & $0.188^{*}$ & $0.250^{*}$ & 0.077 \\
\hline Required content & 0.175 & -0.014 & $0.240^{*}$ & $0.266^{*}$ & 0.120 \\
\hline Obtained content & 0.172 & 0.044 & $0.211^{*}$ & $0.226^{*}$ & 0.113 \\
\hline Guidance skills and effects & 0.055 & -0.046 & 0.084 & $0.189^{*}$ & 0.001 \\
\hline
\end{tabular}

${ }^{*} \mathrm{P}<0.05$.

Table 5. Multiple linear regression analysis of factors influencing the readiness for discharge of patients with prostate cancer surgery.

\begin{tabular}{ccccc}
\hline Item & Regression coefficient & Standard regression coefficient & $t$ & P \\
Constant & 110.432 & - & 4.470 & $<0.001$ \\
Education level & 11.629 & 0.379 & 4.872 & $<0.001$ \\
Monthly family income & 11.407 & 0.157 & 2.173 & 0.032 \\
Place of residence & -9.273 & -0.120 & -1.476 & 0.143 \\
Gleason score & -9.578 & -0.285 & -4.004 & $<0.001$ \\
whether to perform lymphatic dissection & 13.108 & 0.177 & 2.438 & 0.016 \\
whether to discharge with urinary catheter & 22.365 & 0.302 & 4.217 & $<0.001$ \\
Total score of discharge guidance quality & 0.141 & 0.153 & 2.159 & 0.033 \\
\hline
\end{tabular}

The lower middle level.

\section{Discussion}

\subsection{Readiness for Discharge of Patients after Prostate Cancer Surgery}

The results of this study showed that $95.80 \%$ of patients with prostate cancer after surgery were consciously ready to be discharged, and $4.20 \%$ of patients thought they were not ready. The results of this study revealed that the total score and items' average score of discharge readiness for patients with prostate cancer was $147.74 \pm 35.71$ points and $6.13 \pm 1.61$ points (average score of less than 7 indicates inadequate discharge preparation), which are at a the lower middle level level as a whole. This suggests that most patients are not ready for discharge. The total score is higher than the results $(86.48 \pm 10.32$ points) of another domestic study conducted by Liu Huijing et al. [6]. These results could be related to the following factors: 1) The surgical techniques for treating prostate cancer are now relatively mature, and laparoscopy and robotic surgery are mostly utilized; the new surgical techniques result in more precision and less trauma, so the patient can quickly recover and return to daily life. 2) Our department has carried out the concept of ERAS since early time. Patients are less 
stressed during the perioperative period; nursing staff will comprehensively evaluate the patient's condition during the perioperative period, formulate targeted nursing plans, and therefore accelerate the postoperative recovery of patients. 3) Under the influence of Chinese traditional culture, family members will actively help and support each other. Most patients have their family members to accompany and take care of them during hospitalization and after discharge. Good social support provides favorable conditions for patients' recovery.

Judging by the scores of hospital discharge readiness after prostate cancer surgery from high to low, the dimensions were ranked as follows: available social support, coping ability after discharge, disease knowledge and physical condition. The dimensions of patients' own physical condition and disease knowledge received low scores, indicating that the patients were still in their recovery stage rather than fully recovered when discharged from the hospital, and had not yet mastered sufficient disease-related knowledge. This analysis is inconsistent with the research results of other scholars. In the study of Guo Wanpeng et al. [10], patients with aortic dissection presented the highest average score in the dimension of physical condition, followed by coping ability after discharge, disease knowledge, and available social support; in the study of Liu Huijing et al. [6] patients with prostate cancer presented the highest average score in the dimension of disease knowledge, followed by available social support, coping ability after discharge, and physical condition. The reason for the differences could be: the family support system of prostate cancer patients in this study is relatively complete, and thus certain knowledge has been accumulated during the hospitalization process. The relatively complete discharge plan of our department also improves the confidence of the patients on their abilities after discharge, so they respond positively on the coping abilities after discharge. Nevertheless, our department promotes the concept of ERAS, and therefore the average hospital stay of patients with prostate cancer surgery has been significantly shortened. Owing to this, the patients feel that they have insufficient disease knowledge. In addition, patients can be discharged after their physiological functions have basically returned to normal and after they have a certain degree of self-care ability, but the patients are not completely recovered after all, so they often worry about the problems of recovery. Therefore, medical staff shall pay attention to the discharged patients with prostate cancer, provide good discharge guidance and follow-up, to promote the recovery of patients.

\subsection{Quality of Discharge Guidance for Patients after Prostate Cancer Surgery}

Discharge guidance refers to the process during the entire period of the patient's hospitalization where medical staff use interviews or education to let patients and their families comprehend the knowledge of post-discharge self-care. In this study, the quality of patient discharge guidance was at a moderate level $(7.53 \pm$ 1.62), and the difference between the scores of patients' required content (7.05 \pm $2.14)$ and obtained content $(6.94 \pm 2.14)$ was negative, indicating that most pa- 
tients received discharge guidance content that did not meet their expectations. The reasons for the analysis are as follows: patients have high expectations on the nurses, and there is still room for improvement in the level of the nurses' professionalism in discharge guidance; patients' sense of participation in the discharge preparation process is not strong; the forms and content of the nurses' guidance still need to be improved. The dimensional scores of guidance skills and effects were at a relatively high level $(8.06 \pm 1.51)$. In view of the current promotion and application of the concept of ERAS as well as the increase in hospital bed turnover rate, the average hospital stays of patients undergoing radical prostatectomy have been significantly shortened. Under such a medical situation, how to ensure the safety of patients discharged from the hospital and the ability of patients and their families undertaking the follow-up care after discharge have become a problem pending exploration. This suggests that nursing work should also consider various forms of health education, such as mind mapping, multimedia information technology intervention, and ways to obtain information after discharge, etc. It aims to improve the quality of patient health education and increase the readiness and confidence of patients in preparation for discharge.

\subsection{Factors Affecting the Discharge Readiness of Patients after Radical Prostatectomy}

\subsubsection{The Discharge Readiness of Patients after Radical Prostatectomy Is Affected by Education Level and Monthly Family Income}

The results of this study revealed that patients with higher education levels have higher levels of readiness for discharge, which is consistent with the research results of Liu Huijing et al. and Wang Binghua et al. [6] [11]. The study also showed that the higher the family monthly income per capita, the higher the level of the patients' readiness for discharge, which is also consistent with the research results of Liu Huijing et al. [6]. The reason for these results could be that patients with a high level of education have better compliance as well as advanced understanding and acceptance of knowledge, and these patients can obtain disease-related knowledge through multiple channels, such as medical staff, WeChat official accounts, the Internet, books, etc. Therefore, for patients with low education level, the medical staff should focus on strengthening the public education of discharge guidance, paying attention to the feedback of patients, and analyze and adjust the content of the guidance in a timely manner. Patients with lower readiness for discharge usually belong to families with lower economic income; the higher their economic income, the better their readiness for discharge. This could be due to the fact that the long-term follow-up treatment, tumor survival surveillance as well as tumor recurrence monitoring after prostate cancer surgery take a large amount of medical costs, making low-income families face greater economic challenges after discharge from the hospital [12]. Therefore, medical workers should actively understand the needs of cancer patients at various levels, and relevant governmental management departments can also provide patients with better healthcare protection by increasing the propor- 
tion of outpatient expenses included in the medical insurance reimbursement.

\subsubsection{The Discharge Readiness of Patients after Radical Prostatectomy Is Affected by Gleason Score, Lymphatic Dissection, and Discharge with Urinary Catheter}

The results of this study showed that the Gleason score, whether to perform lymphatic dissection and whether to discharge with urinary catheter are important factors influencing the patient's readiness for discharge. The results of Liu Huijing et al. [6] also showed that the Gleason score is an influencing factor of the readiness for discharge of prostate cancer patients, which is consistent with the results of this study. The reason could be as follows: the higher the Gleason score, the severer the patient's symptoms such as urinary incontinence, pain, sexual dysfunction, fatigue, etc. [12]; the ensuing psychological problems, such as low self-esteem, frustration, depression, other emotional reactions and social withdrawal, could also be more obvious [13]. In patients that has undergone lymphatic dissection during the operation, there is a possibility of lymphatic leakage after the operation, which delays the removal of the drainage tube after the operation and prolongs the length of stay in the hospital; the patients lack the knowledge of nursing related to lymphatic leakage, and the ensuing anxiety and nervousness affect their readiness for discharge. Some patients need to be discharged from the hospital with a urinary catheter, yet they lack the knowledge of urinary catheter care; patients may experience urinary incontinence and erectile dysfunction after the catheter is removed, which is extremely harmful to the patients' physiology and psychology, affecting the patients' readiness for discharge.

\subsubsection{The Discharge Readiness of Patients after Radical Prostatectomy Is Affected by the Quality of Discharge Guidance}

The results of this study showed that the discharge readiness score of patients after radical prostatectomy is positively correlated with the discharge guidance quality score, and that the quality of discharge guidance is an important factor influencing the patients' discharge readiness, all indicating that the discharge readiness of patients after radical prostatectomy is related to the quality of discharge guidance. This is consistent with the results of other studies [14] [15]. The content of discharge guidance can provide patients and their families with post-discharge self-care methods, related precautions, medication, rehabilitation training and other medical care information. Guidance skills will affect patients and their families' understanding, acceptance and correct implementation of the guidance content, so high-quality discharge guidance can enhance patients' selfcare ability, reduce disease uncertainty, and improve patients' perception of discharge readiness [16]. Therefore, attention should be paid to the discharge instructions of patients after radical prostatectomy; nurse training should be strengthened to improve the effect of patient discharge guidance; patients with different cultural backgrounds should also be encouraged to participate in the joint decision-making process of their discharge preparation plans. 


\section{Conclusion}

In summary, the discharge readiness of patients after radical prostatectomy and the quality of discharge guidance are at moderate levels and need to be improved. The patient's readiness for discharge is affected by education level, monthly family income, Gleason score, whether to perform lymphatic dissection, whether to discharge with a urinary catheter, and the quality of discharge guidance. Therefore, in clinical work, personalized health education should be implemented according to these influencing factors of hospital discharge readiness, to improve the quality of patient guidance. A comprehensive and effective assessment of the readiness for discharge of prostate cancer patients is the basis for the implementation of discharge management, which can ensure that patients are discharged in the best condition and at the most suitable time, reduce the incidence of complications and re-admission rates after discharge, save medical resources, and also attach particular importance to the self-regulation and management of the patients after discharge.

\section{Fund Project}

Nursing Research Fund of the Third Affiliated Hospital of Sun Yat-sen University (2021HLMS08, 2021HLMS02)

\section{Conflicts of Interest}

The authors declare no conflicts of interest regarding the publication of this paper.

\section{References}

[1] Gu, C.Y., Qing, X.J., Huang, Y.Q., et al. (2019) The Prostate Cancer Precision Screening Program: A Preliminary Report after Recruitment of 2159 Men. National Medical Journal of China, No. 42, 3292-3297.

[2] Ye, D.W. and Zhu, Y. (2015) Epidemiology of Prostate Cancer in China: An Overview and Clinical Implication. Chinese Journal of Surgery, No. 4, 249-252.

[3] Steentjes, L., Siesling, S., Drummond, F.J., van Manen, J.G., Sharp, L. And Gavin, A. (2018) Factors Associated with Current and Severe Physical Side-Effects after Prostate Cancer Treatment: What Men Report. European Journal of Cancer Care, 27, Article ID: e12589. https://doi.org/10.1111/ecc.12589

[4] Wu, H.Y., Lin, H.L., Lin, D.N.,et al. (2021) The Supportive Care Needs of Patients with Prostate Cancer: A Qualitative Study. Chinese Journal of Practical Nursing, 37, 531-537.

[5] Fenwick, A.M. (1979) An Interdisciplinary Tool for Assessing Patients' Readiness for Discharge in the Rehabilitation Setting. Journal of Advanced Nursing, 4, 9-21. https://doi.org/10.1111/j.1365-2648.1979.tb02984.x

[6] Liu, H.j., Liu, W., Dong, J.Q., et al. (2018) Path Analysis of Discharge Readiness and Its Influencing Factors in Elderly Patients with Prostate Cancer after Surgery. National Journal of Andrology, 24, 857-860.

[7] Zhao, H., Feng, X., Yu, R., Gu, D. and Ji, X. (2015) Validation of the Chinese Version of the Readiness for Hospital Discharge Scale on Patients Who Have Undergone 
Laryngectomy. Journal of Nursing Research, 24, 321-328. https://doi.org/10.1097/JNR.0000000000000126

[8] Zhao, H.L., Feng, X.Q.,Yu, R., et al. (2013) Patients' Discharge Readiness for Hospital Discharge: A Systematic Review of Literature. Chinese Nursing Management, 13, 81-83.

[9] Wang, B.H., Wang, H. and Yang, C.Z. (2016) Reliability and Validity of the Chinese Version of the Quality of Discharge Teaching Scale. Chinese Journal of Nursing, 51, 752-755.

[10] Guo, W.P., Zhang, Q. And Li, L. (2018) Influencing Factors of Discharge Readiness for Patients after Aortic Dissection Surgery. Nursing Journal of Chinese People's Liberation Army, 35, 18-22.

[11] Wang, B.H., Wang, H., Yang, C.Z., et al. (2017) Survey of the Readiness for Hospital Discharge and Its Influencing Factors among Coronary Heart Disease Patients. Chinese Journal of Nursing, 52, 138-143.

[12] Fan, X.R., Li, H.Z., Li, Y.Q.,et al. (2013) Long-Term Efficacy of the Treatment for Metastatic Castration-Resistant Prostate Cancer. Chinese Journal of Urology, 34, 885-890.

[13] Qi, J.L., Wang, L.J., Zhou, M.G., et al. (2016) Disease Burden of Prostate Cancer among Men in China, from 1990 to 2013. Chinese Journal of Epidemiology, 37, 778-782.

[14] Zhou, B.W., Zhang, H.M. and Liu, C.H. (2018) Assessing Readiness for Discharge and Associated Factors among COPD Patients. Journal of Nursing Science, 33, 35 38.

[15] Yang, J., He, Y.H., Jiang, L.L., et al. (2018) Analysis of the Status Quo and Influencing Factors of Readiness for Discharge of Patients with Colorectal Cancer Following Enhanced Recovery after Surgery. Chinese Nursing Management, 18, 1617-1621.

[16] Knier, S., Stichler, J.F., Ferber, L. and Catterall, K. (2015) Patients' Perceptions of the Quality of Discharge Teaching and Readiness for Discharge. Rehabilitation Nursing Journal, 40, 30-39. https://doi.org/10.1002/rnj.164 